\title{
Atrial Substrate Underlies the Recurrence after Catheter Ablation in Patients with Atrial Fibrillation
}

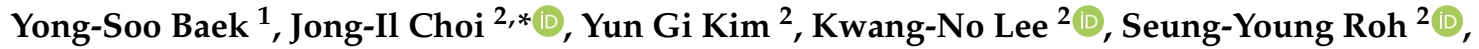 \\ Jinhee Ahn ${ }^{2}$, Dong-Hyeok Kim ${ }^{2}{ }^{\oplus}$, Dae In Lee ${ }^{2}$, Sung Ho Hwang ${ }^{3}{ }^{\circledR}$, Jaemin Shim ${ }^{2}$, \\ Jin Seok Kim ${ }^{2}{ }^{(}$, Dae-Hyeok Kim ${ }^{1}{ }^{1}$, Sang-Weon Park ${ }^{2}$ and Young-Hoon Kim ${ }^{2}$ \\ 1 Division of Cardiology, Department of Internal Medicine, Inha University College of Medicine and Inha \\ University Hospital, Incheon 22212, Korea; existsoo@inha.ac.kr (Y.-S.B.); kdhmd@inha.ac.kr (D.-H.K.) \\ 2 Division of Cardiology, Department of Internal Medicine, Korea University College of Medicine and Korea \\ University Medical Center, Seoul 02841, Korea; tmod0176@gmail.com (Y.G.K.); knlee81@gmail.com (K.-N.L.); \\ rsy008@gmail.com (S.-Y.R.); reinee81@naver.com (J.A.); tomas9912@naver.com (D.-H.K.); \\ acttopia@naver.com (D.I.L.); jshim@korea.ac.kr (J.S.); heartmania@unitel.co.kr (J.S.K.); \\ swparkmd@gmail.com (S.-W.P.); yhkmd@unitel.co.kr (Y.-H.K.) \\ 3 Department of Radiology, Korea University Anam Hospital, Seoul 02841, Korea; sungho77@korea.ac.kr \\ * Correspondence: jongilchoi@korea.ac.kr; Tel.: +82-2-920-5445
}

Received: 14 September 2020; Accepted: 28 September 2020; Published: 30 September 2020

\begin{abstract}
Prediction of recurrences after catheter ablation of atrial fibrillation (AF) remains challenging. We sought to investigate the long-term outcomes after AF catheter ablation. A total of 2221 consecutive patients who underwent catheter ablation for symptomatic AF were included in this study (mean age $55 \pm 11$ years, $20.3 \%$ women, and $59.0 \%$ paroxysmal AF). Extensive ablation, in addition to circumferential pulmonary vein isolation, was more often accomplished in patients with non-paroxysmal AF than in those with paroxysmal AF ( $87.4 \%$ vs. $25.3 \%, p<0.001)$. During a median follow-up of 54 months, sinus rhythm (SR) was maintained in $67.1 \%$ after index procedure. After redo procedures in 418 patients, $83.3 \%$ exhibited SR maintenance. Recurrence rates were similar for single and multiple procedures $(17.4 \%$ vs. $16.7 \%, p=0.765)$. Subanalysis showed that the extent of late gadolinium enhancement (LGE), as assessed by cardiac magnetic resonance, is greater in patients with recurrence than in those without recurrence $(36.2 \pm 23.9 \%$ vs. $21.8 \pm 13.7 \%, p<0.001)$. Cox-regression analysis revealed that non-paroxysmal AF (hazard ratio (HR) 2.238, 95\% confidence interval (CI) 1.905-2.629, $p<0.001$ ), overweight (HR 1.314, 95\% CI 1.107-1.559, $p=0.020$ ), left atrium dimension $\geq 45 \mathrm{~mm}$ (HR 1.284, 95\% CI 1.085-1.518, $p=0.004$ ), AF duration (HR 1.020 per year, 95\% CI 1.006-1.034, $p=0.004$ ), and LGE $\geq 25 \%$ (HR 1.726, 95\% CI 1.330-2.239, $p<0.001$ ) are significantly associated with AF recurrence after catheter ablation. This study showed that repeated catheter ablation improves the clinical outcomes of patients with non-paroxysmal AF, suggesting that AF substrate based on LGE may underpin the mechanism of recurrence after catheter ablation.
\end{abstract}

Keywords: atrial fibrillation; catheter ablation; substrate; magnetic resonance imaging

\section{Introduction}

Atrial fibrillation (AF) is associated with higher rates of mortality, stroke, congestive heart failure, and hospitalization [1]. Given the limited efficacies and potential adverse effects of antiarrhythmic drugs, catheter ablation offers the most effective rhythm control strategy and could be utilized as a first-line treatment in selected patients [2,3]. Reports indicate that in patients with AF progression, multiple procedures may be more effective than single procedures in the long-term [4]. Atrial fibrosis is a major determinant of AF progression; thus, more extensive atrium remodeling favors development of 
persistent arrhythmia [5]. Atrial tissue fibrosis, which is a marker of an underlying substrate, could be visualized with late gadolinium enhancement (LGE) on cardiac magnetic resonance (MR) images and has been shown to be independently associated with the likelihood of recurrent arrhythmia [6]. However, clinical implications of such an association are challenging, and little data are available on the effect of catheter ablation on long-term clinical outcomes with respect to AF progression stratified by AF type and atrial fibrosis as estimated by MR LGE. In this study, we investigated the clinical outcomes of catheter ablation over a 10-year follow-up period. We hypothesized that atrial substrates represented by AF progression underlie the mechanism of recurrence after catheter ablation in patients with AF.

\section{Materials and Methods}

\subsection{Study Population}

A total of 2221 patients who underwent radiofrequency catheter ablation for symptomatic drug-refractory AF between 2004 and January 2016 at a single tertiary hospital were enrolled in this retrospective study. The study flow chart is presented schematically in Figure 1. The exclusion criteria applied were as follows: (1) AF with rheumatic valvular disease; (2) AF refractory to electrical cardioversion; and (3) prior cardiac surgery. In all patients, antiarrhythmic drugs were discontinued for a period of at least five half-lives prior to catheter ablation, and amiodarone was discontinued for at least 4 weeks prior to the procedure. In this study, $\mathrm{AF}$ was defined as paroxysmal if the episodes lasted $<48 \mathrm{~h}$, or terminated spontaneously within 7 days, or if the patients received electric or pharmacological cardioversion within $48 \mathrm{~h}$. AF was defined as non-paroxysmal when AF progressed to persistent (sustained AF (i.e., $>7$ days), or when AF was successfully restored by electrical or pharmacologic cardioversion after $48 \mathrm{~h}$ ), longstanding persistent (continuous AF for $>12$ months), or permanent AF (a decision was made not to restore or maintain sinus rhythm (SR) by any means). The study protocol was approved by the Institutional Review Board of Korea University Anam Hospital Institutional Review Board (2016-AN0210) and complied with the Declaration of Helsinki.

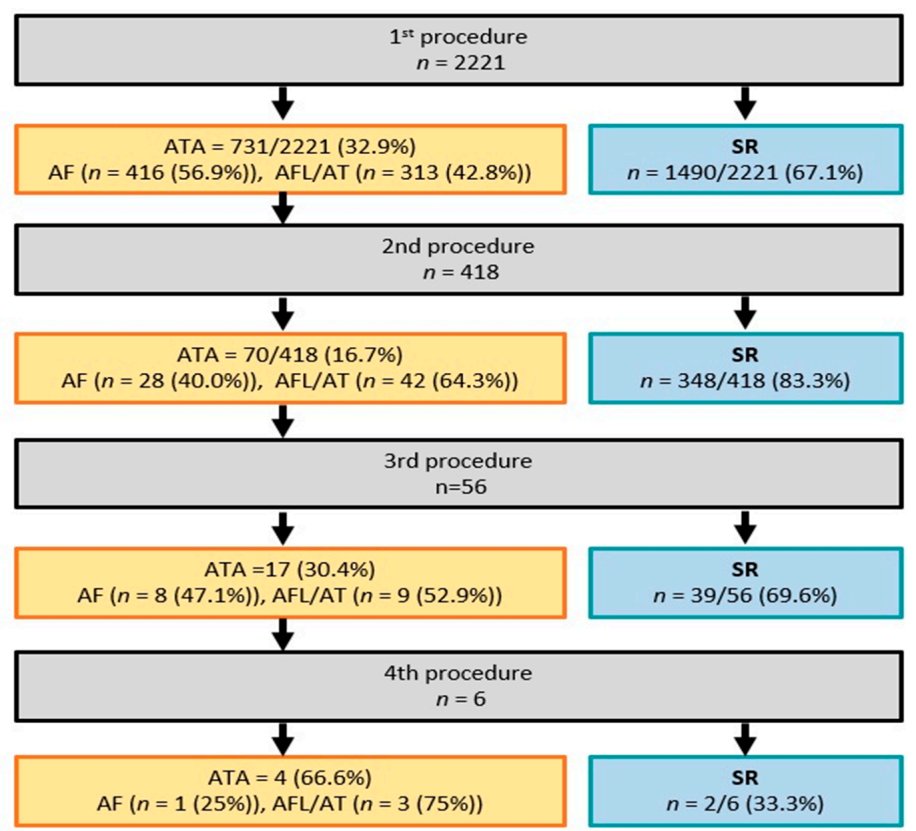

- $\mathrm{ATA}=$ atrial tachyarrhythmia; $\mathrm{AFL}=$ atrial flutter; $\mathrm{AT}=$ atrial tachycardia; $\mathrm{AF}=$ atrial fibrillation; $\mathrm{SR}=$ sinus rhythm

Figure 1. Flow chart of atrial fibrillation (AF) catheter ablation outcomes. 


\subsection{Electrophysiological Studies and Catheter Ablation}

Ablation was performed using radiofrequency energy under three-dimensional (3D) electroanatomical mapping (NavX, St. Jude Medical, Minnetonka, MN, USA; CARTO3, Johnson \& Johnson Inc., Diamond Bar, CA, USA) merged with 3D spiral computed tomography (CT) or cardiac magnetic resonance (CMR). Patients were sedated with intravenous midazolam and fentanyl, and arterial blood gas analysis was performed hourly. Two transseptal punctures were performed using long sheaths (Fast-Cath ${ }^{\mathrm{TM}}$ and Swartz ${ }^{\text {TM }}$ SL1 or 2, St. Jude Medical, AF Division, Minnetonka, MN, USA) and a Brockenbrough needle under fluoroscopic guidance. Unfractionated heparin was administered to maintain an activated clotting time between 300 and $350 \mathrm{~s}$. Radiofrequency energy was delivered at a maximum temperature of $48^{\circ} \mathrm{C}$ and a power of $25-35 \mathrm{~W}$ using a 3.5-mm open irrigated-tip ablation catheter. All patients underwent circumferential pulmonary vein isolation (CPVI). The ablation strategy used was as previously described [7]. During catheter ablation for paroxysmal AF, triggers of sustained AF were evaluated and targeted after CPVI. To identify the triggers initiating paroxysmal AF, multiple electrical cardioversions were subsequently performed in patients with initial AF rhythm under isoproterenol (10-20 $\mu \mathrm{g} / \mathrm{min}$ ) infusion. In patients with SR, AF was induced by burst high right atrial pacing in decrements from $250 \mathrm{~ms}$ to the atrial refractory period. Triggers were identified based on reinitiation of sustained or non-sustained $\mathrm{AF}$ within 2 min of cardioversion. A single premature atrial beat without sustained AF plus atrial tachycardia (AT) was not considered a trigger. During the protocol, a 20-pole circular mapping catheter, an ablation catheter, and a quadripolar catheter were positioned in the left pulmonary vein, right pulmonary vein, and superior vena cava, respectively.

The first beat initiating AF was considered a trigger and the site of origin was analyzed. After CPVI, we repeated the same protocol at least three times to identify non-pulmonary vein focal initiators of AF. The endpoint of the procedure for paroxysmal $\mathrm{AF}$ was the elimination of all triggers, including those of the pulmonary vein (PV). For patients with persistent AF, 3D automated complex fractionated atrial electrography (CFAE) and/or linear ablation, including the roof line, posterior inferior line, perimitral isthmus line, and/or anterior line, was performed at the surgeons' discretion. The endpoint of the procedure for persistent $\mathrm{AF}$ was $\mathrm{AF}$ termination and no induction of sustained atrial arrhythmia. CFAE map settings were as follows: refractory period $49 \mathrm{~ms}$, P-P sensitivity $>0.1 \mathrm{mV}$, and duration $30 \mathrm{~ms}$. If ablation was performed, a CFAE map was used for guidance until AF was terminated or converted to AT or until fractionated activity was eliminated. Areas in the right atrium (RA) based on CFAE were targeted for ablation if AF persisted after extensive left atrium (LA) ablation. If AF was sustained after all RA CFAE had been abolished, a tailored approach was applied on the basis of case-by-case incidence that included LA and/or RA potential-guided focal or linear ablation. If AF was converted to AT during pulmonary vein isolation (PVI) or CFAE-guided ablation, activation and entrainment mapping-guided ablations were performed. Cavotricuspid isthmus (CTI) linear ablation and bidirectional block were performed in patients with clinical atrial flutter. In all patients with persistent AF, the inducibility of sustained AF plus AT by pacing was evaluated when AF and AT were terminated successfully by ablation. If SR was not restored after ablation of the aforementioned lesions, the patients were cardioverted using an internal or external direct-current electrical shock.

\subsection{Post-Ablation Management and Follow-Up}

Ambulatory monitoring and daily 12-lead electrocardiogram (ECG) were applied to patients at 1 to 2 days after AF ablation. Patients were asked to visit our outpatient clinic 1,3, 6, and 12 months after catheter ablation and every 6 months thereafter, or whenever symptoms occurred. Oral anticoagulant and antiarrhythmic medications were continued for at least 3 months after the procedure. The decision to discontinue antiarrhythmic medications after 3 months was made at the physicians' discretion. All patients underwent electrocardiography at every follow-up visit and a 24-h Holter recording was obtained at 3 and 6 months and every 6 months thereafter. However, whenever patients reported palpitations, Holter monitor or event monitor recordings were obtained and evaluated to check for arrhythmia recurrence. We defined AF recurrence as any episode of AF plus AT lasting for $\geq 30 \mathrm{~s}$. 
AF recurrence based on electrocardiography after a blanking period of 3 months (i.e., at $\geq 3$ months post-ablation) was documented as clinical recurrence.

\subsection{Analysis of MR LGE Images}

Cardiac MR examinations were performed using a 3-T MR system (Achieva; Philips Medical Systems, Best, The Netherlands) equipped with a 32-element phased-array cardiac coil. All cardiac MR LGE images were analyzed independently by two experienced radiologists (blinded to the patients' clinical and electrophysiological data) at a commercial software workstation (Terarecon iNtuition; TeraRecon, Foster City, CA, USA). The 3D reconstruction process used to produce MR LGE images was as previously described [8,9]. On transverse or coronal MR LGE images, epicardial and endocardial borders of LA walls were semi-automatically contoured to select the entire LA walls and reconstruct 3D LA models. For quantitative analysis of a selected LA wall, we used signal threshold methods and manual delineation of regions of interest (ROIs) that included, to the greatest possible extent, the normal left ventricular (LV) wall of nulled signal intensity on an MR LGE image. Six and two standard deviation (SD) thresholds above the mean signals of normal LV walls were calculated using ROI means and SD values. Using the six and two SD thresholds, LA wall substrates were classified into three groups: (1) fibrotic substrate with a signal $\geq 6$ SD threshold; (2) intermediate substrate with a signal $\geq 2$ SD threshold but $<6$ SD threshold; and (3) normal substrate with a signal $<2$ SD threshold. In addition, to better delineate LA wall composition, colored look-up table masks were applied to 3D LA models as follows: yellow for fibrotic substrate, gray for indeterminate substrate, and blue for normal substrate. Figure 2 shows two examples of processed 3D reconstructions of cases with low and high MR LGE at LA walls.

A.

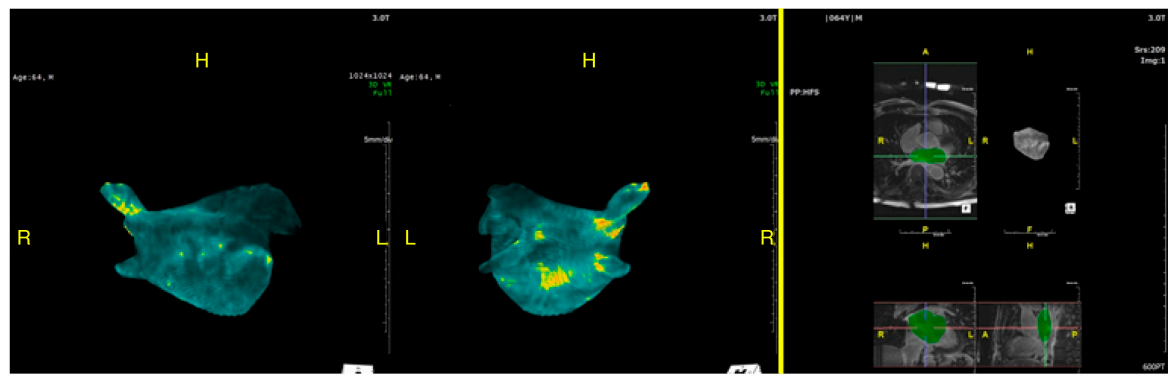

Sixty four year-old male patients with paroxysmal AF. The extent of LA-LGE was $8 \%$ of whole LA wall surface

B.

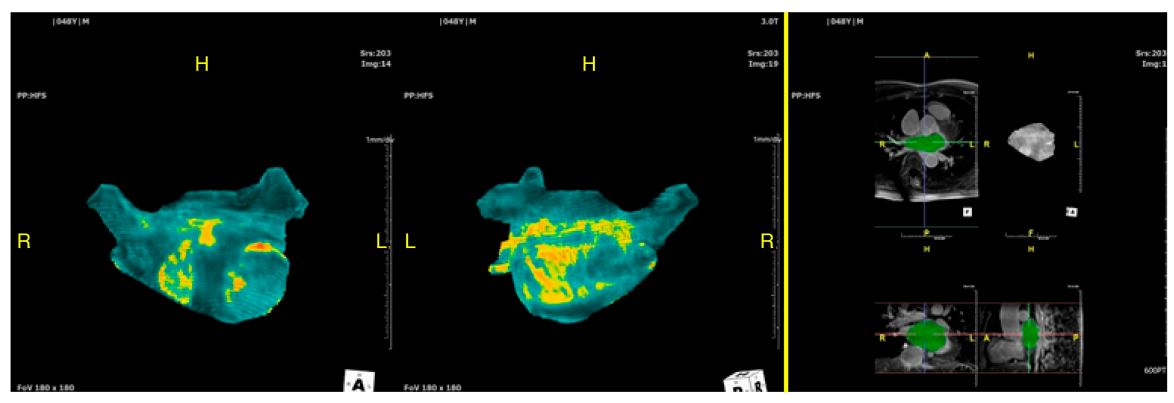

Forty eight year-old male patients with persistent AF. The extent of LA-LGE was $41 \%$ of whole LA wall surface

Figure 2. Processed 3D reconstructions showing low and high late gadolinium enhancement in cardiac magnetic resonance images (MR LGE) within left atrium (LA) walls. (A) A 64-year-old man with paroxysmal AF. (B) A 48-year-old man with persistent AF.

\subsection{Statistical Analysis}

Results are presented as means \pm SD for continuous variables and as proportions for categorical variables. For patients with or without clinical recurrence, continuous variables were compared using 
Student's $t$-test, and categorical variables were analyzed using the chi-square test. The $p$ values less than 0.05 were regarded as statistically noticeable in our study. Times to AF plus AT recurrence were calculated by Kaplan-Meier analysis, and comparisons were performed using log-rank statistics. Univariate regression analysis was used to identify relationships between clinical variables and AF/AT recurrences after index ablation. Multivariate logistic regression analysis was performed using variables with $p$ values $<0.05$ in the univariate analysis to identify predictors of clinical recurrence following ablation. Statistical analysis was performed using SPSS software version 24.0 (IBM, Armonk, NY, USA).

\section{Results}

\subsection{Patient Characteristics}

Baseline patient characteristics are presented in Table 1. The mean age of the 2221 subjects was $55.1 \pm 10.8$ years, and $20.3 \%$ were female. The mean antero-posterior LA diameter and LV ejection fraction, which were determined using echocardiographic findings, were $41.2 \pm 6.1 \mathrm{~mm}$ and $55.1 \pm 5.8 \%$, respectively (Table 2).

Table 1. Baseline clinical characteristics of study patients according to clinical recurrence after catheter ablation.

\begin{tabular}{|c|c|c|c|c|}
\hline & Overall & $\begin{array}{c}\text { No Recurrence } \\
\text { Group }\end{array}$ & $\begin{array}{c}\text { Recurrence } \\
\text { Group }\end{array}$ & \multirow{2}{*}{${ }^{*} p$ Value } \\
\hline & $(n=2221)$ & $(n=1490)$ & $(n=731)$ & \\
\hline Age (years) & $55.1 \pm 10.8$ & $55.0 \pm 10.8$ & $56.5 \pm 10.7$ & 0.004 \\
\hline Female, $n(\%)$ & $451(20.3)$ & $303(20.3)$ & $148(20.2)$ & 0.961 \\
\hline Non-paroxysmal AF, $n(\%)$ & $911(41.0)$ & $487(32.7)$ & $424(58.0)$ & $<0.001$ \\
\hline Height, $\mathrm{cm}$ & $168.2 \pm 8.2$ & $168.0 \pm 8.0$ & $168.5 \pm 8.1$ & 0.269 \\
\hline Weight, kg & $70.8 \pm 11.4$ & $70.4 \pm 11.6$ & $71.7 \pm 11.1$ & 0.040 \\
\hline $\mathrm{BSA}, \mathrm{m}^{2}$ & $1.8 \pm 0.4$ & $1.8 \pm 0.5$ & $1.8 \pm 0.2$ & 0.927 \\
\hline BMI, $\mathrm{kg} / \mathrm{m}^{2}$ & $25.1 \pm 3.4$ & $24.9 \pm 3.5$ & $25.3 \pm 3.2$ & 0.078 \\
\hline $\mathrm{HF}, n(\%)$ & $320(14.4)$ & $193(13.0)$ & $127(17.4)$ & 0.005 \\
\hline Hypertension, $n(\%)$ & $732(33.0)$ & 467 (31.3) & $265(36.3)$ & 0.021 \\
\hline $\mathrm{DM}, n(\%)$ & $171(7.7)$ & $103(6.9)$ & $68(9.3)$ & 0.047 \\
\hline Stroke/TIA, $n(\%)$ & $116(5.2)$ & $72(4.8)$ & $44(6.0)$ & 0.237 \\
\hline Vascular disease, $n(\%)$ & $104(4.7)$ & $76(5.1)$ & $28(3.8)$ & 0.200 \\
\hline $\mathrm{CHA}_{2} \mathrm{DS}_{2}$-VASc score & $1.15 \pm 1.22$ & $1.09 \pm 1.20$ & $1.28 \pm 1.24$ & $<0.001$ \\
\hline \multicolumn{5}{|l|}{ Post-ABL medication } \\
\hline Beta-blocker, $n(\%)$ & $403(18.4)$ & $268(18.2)$ & $248(34.3)$ & $<0.001$ \\
\hline Statin, $n(\%)$ & $516(23.5)$ & $224(15.2)$ & $179(24.8)$ & $<0.001$ \\
\hline $\mathrm{AAD}, n(\%)$ & $342(15.6)$ & $192(13.0)$ & $150(20.7)$ & $<0.001$ \\
\hline \multicolumn{5}{|l|}{ Laboratory findings } \\
\hline BUN (mg/dL) & $15.7 \pm 4.8$ & $15.7 \pm 4.9$ & $15.6 \pm 4.5$ & 0.733 \\
\hline Creatinine (mg/dL) & $1.0 \pm 0.4$ & $1.0 \pm 0.4$ & $1.0 \pm 0.2$ & 0.381 \\
\hline Total cholesterol (mg/dL) & $179.7 \pm 37.1$ & $180.3 \pm 35.9$ & $177.8 \pm 40.4$ & 0.419 \\
\hline HDL cholesterol (mg/dL) & $48.2 \pm 11.8$ & $49.3 \pm 12.3$ & $49.2 \pm 12.2$ & 0.843 \\
\hline LDL cholesterol (mg/dL) & $108.0 \pm 30.5$ & $109.0 \pm 30.4$ & $105.9 \pm 30.4$ & 0.053 \\
\hline Triglyceride (mg/dL) & $136.3 \pm 83.9$ & $136.3 \pm 86.1$ & $136.3 \pm 79.1$ & 0.996 \\
\hline $\mathrm{HbA} 1 \mathrm{c}$ & $5.9 \pm 0.8$ & $5.9 \pm 0.8$ & $5.9 \pm 0.7$ & 0.465 \\
\hline AF duration, years & $4.6 \pm 4.7$ & $4.2 \pm 4.4$ & $5.3 \pm 5.2$ & $<0.001$ \\
\hline
\end{tabular}

* As determined by Student's $t$-test or the chi-square test for recurrence vs. no recurrence. AAD, antiarrhythmic drug at $\mathrm{AF}$ recurrence; $\mathrm{ABL}$, ablation; $\mathrm{AF}$, atrial fibrillation; $\mathrm{BMI}$, body mass index; $\mathrm{BSA}$, body surface area; $\mathrm{BUN}$, blood urea nitrogen; $\mathrm{CHA}_{2} \mathrm{DS}_{2}$, congestive heart failure, hypertension, age $=75$ years, diabetes mellitus, stroke; $\mathrm{DM}$, diabetes mellitus; Hb1Ac, glycated hemoglobin; HDL, high-density lipoprotein; HF, heart failure; LDL, low-density lipoprotein; TIA, transient ischemic attack; and VASc, vascular disease, age 65-75 years, sex category. 
Table 2. Echocardiographic and ablation findings.

\begin{tabular}{|c|c|c|c|c|}
\hline & Overall & $\begin{array}{c}\text { No Recurrence } \\
\text { Group }\end{array}$ & $\begin{array}{c}\text { Recurrence } \\
\text { Group }\end{array}$ & \multirow{2}{*}{${ }^{*} p$ Value } \\
\hline & $(n=2221)$ & $(n=1490)$ & $(n=731)$ & \\
\hline \multicolumn{5}{|l|}{ Echocardiographic findings } \\
\hline LA dimension $(\mathrm{mm})$ & $41.2 \pm 6.1$ & $40.4 \pm 5.9$ & $42.8 \pm 6.1$ & $<0.001$ \\
\hline LV ejection fraction (\%) & $55.1 \pm 5.8$ & $55.4 \pm 5.6$ & $54.4 \pm 6.3$ & 0.001 \\
\hline LA dimension $\geq 45 \mathrm{~mm}(\%)$ & $519(23.6)$ & $277(18.8)$ & $242(33.3)$ & $<0.001$ \\
\hline \multicolumn{5}{|l|}{ Ablation findings } \\
\hline Total ablation time (min) & $114 \pm 54$ & $102 \pm 47$ & $129 \pm 59$ & $<0.001$ \\
\hline Total fluoroscopy time (min) & $68 \pm 34$ & $63 \pm 32$ & $76 \pm 36$ & $<0.001$ \\
\hline Total procedure time (min) & $275 \pm 108$ & $252 \pm 98$ & $309 \pm 113$ & $<0.001$ \\
\hline \multicolumn{5}{|l|}{ Ablation lesion set } \\
\hline CPVI, $n(\%)$ & $2221(100)$ & $1490(100)$ & $731(100)$ & NA \\
\hline CTI, $n(\%)$ & $1425(64.0)$ & $907(61.0)$ & $518(71.0)$ & $<0.001$ \\
\hline CFAE, $n(\%)$ & $507(22.8)$ & $288(19.3)$ & $219(30.0)$ & $<0.001$ \\
\hline Linear ablation, $n(\%)$ & $268(12.1)$ & $131(8.8)$ & $137(18.7)$ & $<0.001$ \\
\hline Biatrial ablation, $n(\%)$ & $520(23.0)$ & $236(16.0)$ & $284(39.0)$ & $<0.001$ \\
\hline
\end{tabular}

The proportions of non-paroxysmal $\operatorname{AF}(p<0.001)$, heart failure $(p=0.005)$, hypertension $(p=0.021)$, and diabetes mellitus $(p=0.047)$ were significantly greater in patients with clinical recurrence than in those without. Preprocedural echocardiograms showed that mean LA dimension was larger $(p<0.001)$ and mean LV ejection fraction was lower $(p<0.001)$ in patients with recurrence. Furthermore, mean $\mathrm{AF}$ duration $(p<0.001)$, mean $\mathrm{CHA}_{2} \mathrm{DS}_{2}$-VASc score $(p<0.001)$, and mean body weight $(p=0.04)$ were greater in patients with clinical recurrence.

\subsection{Radiofrequency Catheter Ablation: Index and Repeat Procedures}

All CPVIs were successfully achieved during the first procedure. A total of 1425 patients $(64.0 \%)$ underwent CTI ablation with bidirectional block. CFAE and linear ablation were performed in 507 $(22.8 \%)$ and $268(12.1 \%)$ patients, respectively, and $520(23.0 \%)$ patients had biatrial ablation in the LA and RA, as previously described. Clinical outcomes after ablation are summarized in Figure 1. During a median follow-up period of 54 months after the index procedure, SR was maintained in 1490 of the 2221 study subjects (67.1\%). Of the 731 patients diagnosed with clinical recurrence after the index procedure, $416(56.9 \%)$ had recurrence of AF or atrial flutter and $313(42.8 \%)$ had AT recurrence. Second, third, and fourth procedures were performed in 418, 70, and 4 patients, respectively, of the 731 patients, and the corresponding rates of SR maintenance were $83.3 \%, 69.6 \%$, and $33.3 \%$ after the repeat procedures. The mean procedure time for the 2221 study subjects was $275 \pm 108 \mathrm{~min}$, the mean ablation time was $114 \pm 54 \mathrm{~min}$, and the mean total ablation and procedure times were $114 \pm 54$ and $275 \pm 108 \mathrm{~min}$, respectively (Table 2).

\subsection{Relationship between MR LGE and Recurrence}

Representative MR LGE findings in patients with or without recurrence are provided in Figure 2. Preprocedural MR imaging was performed to evaluate the LA and PVs in 405 patients before catheter ablation. Patients who experienced recurrence had a significantly greater mean LGE area percentage than those who did not experience recurrence $(36.2 \pm 23.9 \%$ vs. $21.8 \pm 13.7 \%, p<0.001)$ (Figure 3). 


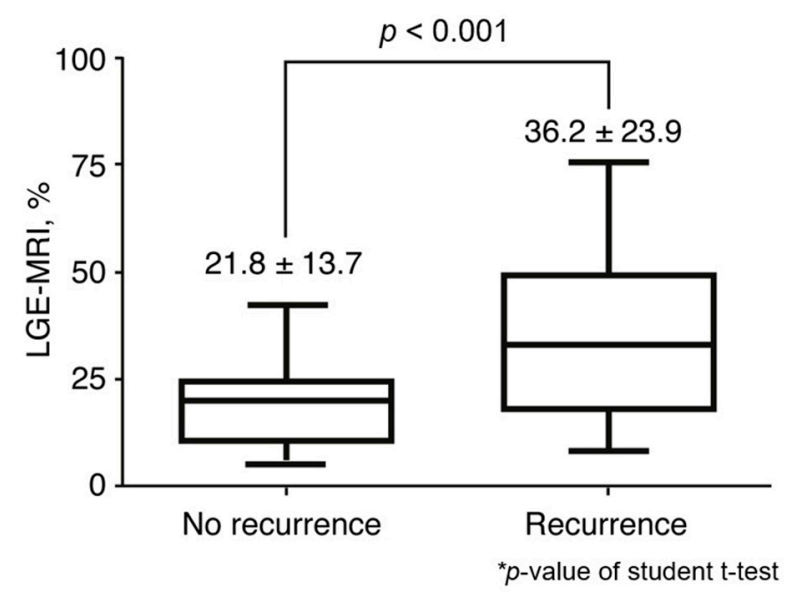

Figure 3. Comparison of MR LGE burdens between patients with and those without clinical recurrence after catheter ablation.

\subsection{Predictors of Clinical Recurrence}

Univariate and multivariate analyzes were performed to identify predictors of clinical recurrence (Table 3). Multivariate model 1, which was adjusted for relevant baseline risks, showed that non-paroxysmal AF (hazard ratio (HR) 2.254, 95\% confidence interval (CI) 1.919-2.648, $p<0.001$ ), overweight/obesity (HR 1.391, 95\% CI 1.176-1.937, $p=0.018$ ), LA dimension $\geq 45 \mathrm{~mm}$ (HR 1.279, 95\% CI 1.005-1.033, $p=0.004$ ), and AF duration (HR 1.019, 95\% CI 1.005-1.033, $p=0.008$ ) are independently associated with clinical recurrence. In multivariate model 2, which included MR LGE and was adjusted for relevant baseline risks, an MR LGE area percentage $\geq 25 \%$ independently predicted clinical recurrence (HR 1.726, 95\% CI 1.330-2.239, $p<0.001$ ). 
Table 3. Clinical predictors of atrial fibrillation recurrence after catheter ablation.

\begin{tabular}{|c|c|c|c|c|c|c|c|c|c|}
\hline & \multicolumn{3}{|c|}{ Univariate } & \multicolumn{3}{|c|}{ Multivariate Model 1 * } & \multicolumn{3}{|c|}{ Multivariate Model $2+$} \\
\hline & HR & $95 \% \mathrm{CI}$ & $p$ Value & HR & $95 \% \mathrm{CI}$ & $p$ Value & HR & $95 \% \mathrm{CI}$ & $p$ Value \\
\hline Age & 1.013 & $1.006-1.020$ & $<0.001$ & 1.007 & $1.000-1.015$ & 0.051 & 1.008 & $1.000-1.015$ & 0.043 \\
\hline Female & 0.978 & $0.817-1.172$ & 0.810 & 1.013 & $0.840-1.222$ & 0.890 & 1.013 & $0.840-1.222$ & 0.893 \\
\hline Non-paroxysmal AF & 2.587 & $2.231-2.999$ & $<0.001$ & 2.254 & $1.919-2.648$ & $<0.001$ & 2.238 & $1.905-2.629$ & $<0.001$ \\
\hline Overweight or obese & 1.216 & $1.006-1.470$ & 0.043 & 1.391 & $1.176-1.937$ & 0.018 & 1.314 & $1.107-1.559$ & 0.020 \\
\hline $\mathrm{HF}$ & 1.355 & $1.119-1.641$ & 0.002 & 1.070 & $0.879-1.302$ & 0.499 & 1.076 & $0.884-1.310$ & 0.464 \\
\hline HTN & 1.212 & $1.042-1.410$ & 0.013 & 1.076 & $0.917-1.264$ & 0.777 & 1.068 & $0.910-1.254$ & 0.421 \\
\hline $\mathrm{DM}$ & 1.325 & $1.032-1.700$ & 0.027 & 1.231 & $0.955-1.588$ & 0.109 & 1.240 & $0.962-1.599$ & 0.097 \\
\hline LA dimension $\geq 45 \mathrm{~mm}$ & 1.918 & $1.646-2.234$ & $<0.001$ & 1.279 & $1.005-1.033$ & 0.004 & 1.284 & $1.085-1.518$ & 0.004 \\
\hline MR LGE $\geq 25 \%$ & 1.022 & $1.014-1.031$ & $<0.001$ & & & & 1.726 & $1.330-2.239$ & $<0.001$ \\
\hline AF duration, per year & 1.017 & $1.003-1.031$ & 0.017 & 1.019 & $1.005-1.033$ & 0.008 & 1.020 & $1.006-1.034$ & 0.004 \\
\hline
\end{tabular}

* Adjusted for age, sex, persistent AF (stepwise model (covariates: age, sex, non-paroxysmal AF, overweight, HF, HTN, DM, LA dimension, and AF duration)); + Adjusted for age, sex, persistent AF (stepwise model (covariates: age, sex, non-paroxysmal AF, overweight, HF, HTN, DM, LA dimension, AF duration, and MR LGE $\geq 25 \%$ )); $\mathrm{AF}$, atrial fibrillation; CI, confidence interval; DM, diabetes mellitus; HF, heart failure; HTN, hypertension; LA, left atrium; and MR LGE, late gadolinium enhancement in cardiac magnetic resonance images. 


\subsection{Effect of Multiple Procedures According to AF Type}

During a mean follow-up of $57.2 \pm 37.9$ months, SR was maintained in $76.6 \%$ of patients with paroxysmal AF and in 53.5\% of those with non-paroxysmal AF (log rank $p<0.001$, Figure $4 \mathrm{~A})$. Kaplan-Meier estimates of AF plus AT-free survival rates showed that the recurrence rates were similar between patients who underwent single and those who had multiple procedures ( $\log \operatorname{rank} p=0.863$, Figure 4B). Kaplan-Meier analyzes according to AF type and number of procedures showed that multiple and single procedures have similar AF plus AT-free survival rates in patients with paroxysmal $\mathrm{AF}(\log \operatorname{rank} p=0.661)$. Patients with non-paroxysmal AF who received multiple procedures had a significantly lower recurrence rate after the last procedure than those who underwent a single procedure (log rank $p=0.022)$ (Figure 5).

A.

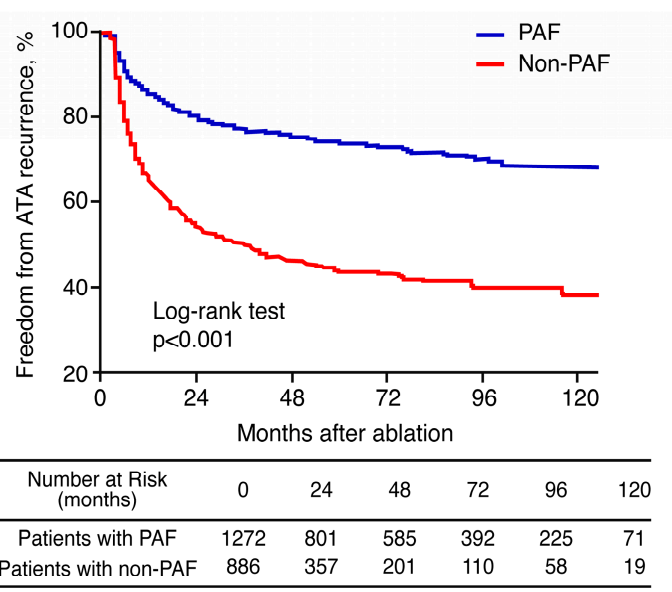

B.

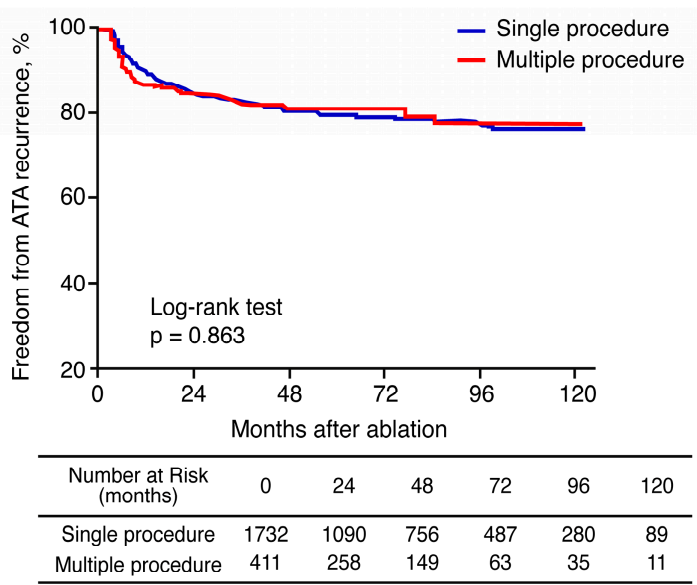

Figure 4. Kaplan-Meier curves showing (A) cumulative AF-free survival rates in patients with paroxysmal AF and non-paroxysmal AF and (B) AF-free survival rates in patients who underwent single and those who had multiple procedures.

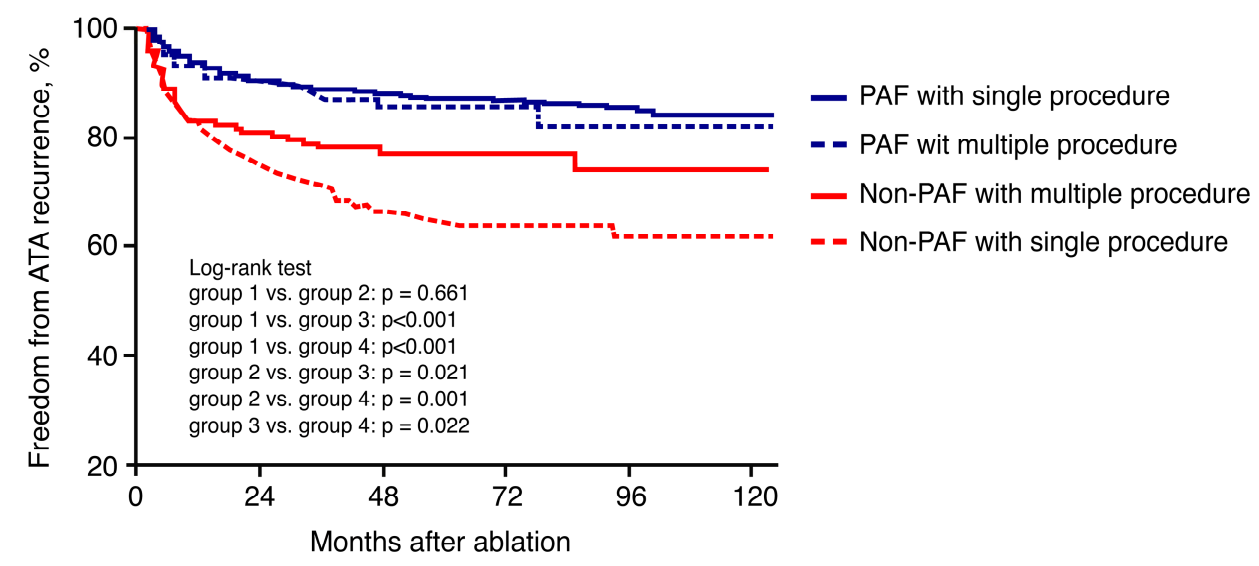

\begin{tabular}{lcccccc}
\hline $\begin{array}{c}\text { Number at Risk } \\
\text { (months) }\end{array}$ & 0 & 24 & 48 & 72 & 96 & 120 \\
\hline Group 1 (PAF with single procedure) & 1092 & 759 & 565 & 383 & 223 & 70 \\
Group 2 (PAF with multiple procedure) & 170 & 118 & 74 & 30 & 18 & 10 \\
Group 3 (Non-PAF with single procedure) & 640 & 331 & 191 & 104 & 64 & 19 \\
Group 4 (Non-PAF with multiple procedure) & 241 & 140 & 75 & 35 & 18 & 3 \\
\hline
\end{tabular}

Figure 5. Kaplan-Meier curves according to AF type and number of procedures. 


\section{Discussion}

\subsection{Main Findings}

This study provides long-term clinical outcome data (over a 10-year follow-up period) of patients with AF who underwent catheter ablation. The main findings of the study are as follows: (1) patients who experienced recurrence were more likely to have hypertension, diabetes, a higher $\mathrm{CHA}_{2} \mathrm{DS}_{2}-\mathrm{VASc}$ score, and heart failure; (2) multiple and single procedures have similar recurrence rates in patients with paroxysmal AF, and multiple procedures have better clinical outcomes than single procedures in those with non-paroxysmal AF; (3) recurrence rates were significantly higher in patients with non-paroxysmal AF during the long-term follow-up; (4) cardiac MR data showed that patients with recurrence had increased LGE; and (5) LGE $\geq 25 \%$, non-paroxysmal AF, overweight/obesity, and AF duration were associated with clinical recurrence.

\subsection{Long-Term Clinical Outcomes According to AF Type}

Ouyang et al. reported a stable SR in $46.6 \%$ of patients after a single CPVI procedure and in $79.5 \%$ of patients with paroxysmal AF after multiple procedures, and progression toward chronic AF in only $2.4 \%$ of the patients after a median follow-up of 4.6 years [10]. Previous studies have reported success rates of 56-58.7\% after index CPVI in patients with paroxysmal AF, which increased to 87-89.4\% after multiple procedures [3,11]. In patients with persistent AF, 59\% of patients assigned to a PVI alone group were free of recurrent AF, whereas $49 \%$ of patients assigned to a PVI plus CFAE group and $46 \%$ of patients assigned to a PVI plus linear ablation group remained free of $\operatorname{AF}(p=0.15)$ [12]. Scherr et al. investigated the long-term outcomes of 150 patients treated using a stepwise ablation approach for persistent AF and found arrhythmia-free survival rates of $35.3 \pm 3.9,28.0 \pm 3.7$, and $16.8 \pm 3.2 \%$ at, respectively, 1, 2, and 5 years after a single procedure [13]. A meta-analysis demonstrated that a single AF procedure resulted in freedom from atrial arrhythmia over long-term follow-up in 53.1\% (95\% CI $46.2-60.0 \%)$ of all patients, $54.1 \%$ (95\% CI 44.4-63.4\%) of patients with paroxysmal AF, and $41.8 \%(95 \%$ CI 25.2-60.5\%) of patients with non-paroxysmal AF. Moreover, the long-term overall success rate after multiple procedures was 79.8\% (95\% CI 75.0-83.8\%) [4].

AF is a progressive disease associated with structural remodeling, which leads to a gradual increase in AF burden [2]. Recently, Tilz et al. reported a rate of progression to persistent AF of only 6.2\% over a 10-year follow-up after catheter ablation and suggested that CPVI may delay or prevent AF progression [3]. According to our findings, patients with paroxysmal AF had better clinical outcomes than those with non-paroxysmal AF, and those with non-paroxysmal AF who underwent multiple procedures had a lower recurrence rate than those who had a single procedure. These findings strongly suggest that in non-paroxysmal AF, multiple procedures and early intervention (such as catheter ablation in paroxysmal AF) play an important role in preventing progression to the irreversible stage.

\subsection{Clinical Predictors of SR Maintenance after Catheter Ablation}

AF ablation is associated with various recurrence rates, mostly due to patient-specific preprocedural factors and specific procedural factors [14]. Predictors of recurrence after AF ablation divided depending on underling structural, electrical, and autonomic substrate [14,15]. In this study, age, non-paroxysmal AF, overweight/obesity, hypertension, diabetes mellitus, LA diameter, MR LGE, and AF duration were found to be associated with AF recurrence by univariate analysis. Previous studies suggested that multiple factors, including persistent AF, AF duration, LA diameter, presence of underlying cardiomyopathy, and previous episodes of congestive heart failure influence long-term follow-up efficacy of radiofrequency catheter ablation in AF [16]. Much evidence indicates that overweight/obesity is associated with the development of AF [17]. A high body mass index has also been shown to be associated with an increased risk of AF recurrence after catheter ablation and progression from paroxysmal to permanent AF [18], and it has also been reported that delayed AF recurrence after catheter ablation is associated with the risk of components of metabolic syndrome, such as hypertension, 
diabetes mellitus, and overweight status [19]. Atrial fibrosis is a condition that involves an underpinned underlying substrate and could be visualized on cardiac MR images, which could also be used to individualize catheter ablation strategies. In a previous study, atrial tissue fibrosis, as estimated by delayed enhancement on MR images, was reported to be independently associated with the risk of recurrent arrhythmia in patients with AF after catheter ablation [6].

\subsection{Effect of Atrial Substrate on Clinical Recurrence}

In this study, patients with AF recurrence after catheter ablation exhibited more LA remodeling and fibrosis based on echocardiography and MR LGE. In patients with AF, atrial fibrosis is a major determinant of the success of rhythm control strategies, including catheter ablation $[6,20]$. Delayed enhancement on MR images is a known marker of fibrotic non-viable ventricular myocardium [20], and atrial fibrosis stratified by MR LGE may aid in selection of appropriate candidates and optimal strategies for catheter ablation of AF [21]. Therapeutic approaches that utilize delayed-enhancement MR imaging for detection of left atrial fibrosis provide a means of enhancing the tailored management of AF [22]. In our study, we observed that an MR LGE $\geq 25 \%$ is strongly associated with clinical recurrence after AF ablation during long-term follow-up. This finding is consistent with the findings of the Delayed-Enhancement MRI Determinant of Successful Radiofrequency Catheter Ablation of Atrial Fibrillation (DECAAF) study, in which atrial fibrosis, as estimated by delayed enhancement on MR images, was found to be independently associated with the likelihood of recurrent arrhythmia [6]. MR LGE to identify the atrial substrate is important in two aspects: one is that it can avoid unnecessary AF ablation procedures, and the other is that it can be feasible and effective for targeting re-entrant drivers or anatomic MRI LGE detected gaps in the AF ablation strategy [23,24]. Therefore, our results indicate that atrial fibrosis, as estimated using MR LGE, may offer a robust means of determining optimal AF management and deciding upon preemptive strategies to prevent AF progression. In the longer term, efforts to identify atrial substrate using MR LGE would allow more patient-centered intervention and patient-tailored decision-making for better clinical outcome in patients undergoing AF ablation.

\subsection{Study Limitations}

This study has several limitations that warrant consideration. First, this study is inherently limited by its non-randomized, retrospective, single center registry-based design. Second, ECG monitoring and 24-h Holter ECG might have missed asymptomatic episodes of AF. Third, cardiac MR was performed in only 405 of the 2221 patients because it was conducted on consecutive patients from 2013. Fourth, we did not perform MR LGE after AF ablation to evaluate how the fibrotic substrate alters before and after the procedure.

\section{Conclusions}

In conclusion, this long-term follow-up study showed that catheter ablation is an effective strategy for rhythm control in patients with non-paroxysmal AF requiring multiple procedures and in those with paroxysmal AF. Atrial substrate, as estimated by MR LGE, is independently associated with clinical outcome after catheter ablation of $\mathrm{AF}$, suggesting that it is an underlying factor of AF recurrence.

Author Contributions: Conceptualization, Y.-S.B., J.-I.C. and Y.-H.K.; Data curation, Y.G.K., K.-N.L., S.-Y.R., J.A., D.-H.K. (Dong-Hyeok Kim), D.I.L., S.H.H., J.S., J.S.K., D.-H.K. (Dae-Hyeok Kim) and S.-W.P.; Formal analysis, Y.-S.B.; Investigation, Y.-S.B., J.-I.C.; Methodology, Y.-S.B., J.-I.C.; Supervision, J.-I.C. and Y.-H.K.; Validation, Y.-S.B.; Writing—original draft, Y.-S.B.; Writing—review \& editing, Y.-S.B. and J.-I.C. All authors have contributed significantly to the article. All authors have read and agreed to the published version of the manuscript. 
Funding: This research was funded by a grant from Korea University (J.-I.C.), Korea University Anam Hospital (J.-I.C.), Inha University (Y.-S.B.), and Inha University Hospital (Y.-S.B.); by grants from the Basic Science Research Program through the National Research Foundation of Korea funded by the Korean Ministry of Education (NRF-2015R1D1A1A02061859 to J.-I.C); by the Korean Ministry of Science, ICT \& Future Planning (NRF-2012R1A1A1013260 to J.-I.C); by the Bio \& Medical Technology Development Program of the National Research Foundation, and by the Korean government (Ministry of Science and ICT) (2019M3E5D1A02069617 to Y.-S.B.).

Conflicts of Interest: The authors declare no conflict of interest. The funders had no role in the design of the study; in the collection, analyzes, or interpretation of data; in the writing of the manuscript, or in the decision to publish the results.

\section{References}

1. Kirchhof, P.; Benussi, S.; Kotecha, D.; Ahlsson, A.; Atar, D.; Casadei, B.; Castellà, M.; Diener, H.-C.; Heidbuchel, H.; Hendriks, J.; et al. 2016 ESC Guidelines for the management of atrial fibrillation developed in collaboration with EACTS. Eur. Hear. J. 2016, 37, 2893-2962. [CrossRef]

2. January, C.T.; Wann, L.S.; Alpert, J.S.; Calkins, H.; Cigarroa, J.E.; Cleveland, J.C.; Conti, J.B.; Ellinor, P.T.; Ezekowitz, M.D.; Field, M.E.; et al. 2014 AHA/ACC/HRS Guideline for the Management of Patients with Atrial Fibrillation. J. Am. Coll. Cardiol. 2014, 64, e1-e76. [CrossRef]

3. Tilz, R.R.; Heeger, C.-H.; Wick, A.; Saguner, A.M.; Metzner, A.; Rillig, A.; Wohlmuth, P.; Reissmann, B.; Lemes, C.; Maurer, T.; et al. Ten-Year Clinical Outcome After Circumferential Pulmonary Vein Isolation Utilizing the Hamburg Approach in Patients with Symptomatic Drug-Refractory Paroxysmal Atrial Fibrillation. Circ. Arrhythm. Electrophysiol. 2018, 11, e005250. [CrossRef]

4. Ganesan, A.N.; Shipp, N.J.; Brooks, A.G.; Kuklik, P.; Lau, D.H.; Lim, H.S.; Sullivan, T.; Roberts-Thomson, K.C.; Sanders, P. Long-term Outcomes of Catheter Ablation of Atrial Fibrillation: A Systematic Review and Meta-analysis. J. Am. Hear. Assoc. 2013, 2. [CrossRef] [PubMed]

5. Smith, J.G.; Newton-Cheh, C.; Almgren, P.; Struck, J.; Morgenthaler, N.G.; Bergmann, A.; Platonov, P.G.; Hedblad, B.; Engström, G.; Wang, T.J.; et al. Assessment of Conventional Cardiovascular Risk Factors and Multiple Biomarkers for the Prediction of Incident Heart Failure and Atrial Fibrillation. J. Am. Coll. Cardiol. 2010, 56, 1712-1719. [CrossRef]

6. Marrouche, N.F.; Wilber, D.; Hindricks, G.; Jais, P.; Akoum, N.; Marchlinski, F.; Kholmovski, E.; Burgon, N.; $\mathrm{Hu}, \mathrm{N}$.; Mont, L.; et al. Association of Atrial Tissue Fibrosis Identified by Delayed Enhancement MRI and Atrial Fibrillation Catheter Ablation. JAMA 2014, 311, 498-506. [CrossRef] [PubMed]

7. Lee, K.-N.; Roh, S.-Y.; Baek, Y.-S.; Park, H.-S.; Ahn, J.; Kim, D.-H.; Lee, D.I.; Shim, J.; Choi, J.-I.; Park, S.-W.; et al. Long-Term Clinical Comparison of Procedural End Points After Pulmonary Vein Isolation in Paroxysmal Atrial Fibrillation. Circ. Arrhythm. Electrophysiol. 2018, 11. [CrossRef] [PubMed]

8. Hwang, S.H.; Oh, Y.-W.; Lee, D.I.; Shim, J.; Park, S.-W.; Kim, Y.-H. Relation between left atrial wall composition by late gadolinium enhancement and complex fractionated atrial electrograms in patients with persistent atrial fibrillation: Influence of non-fibrotic substrate in the left atrium. Int. J. Cardiovasc. Imaging 2015, 31, 1191-1199. [CrossRef]

9. Kim, Y.G.; Shim, J.; Oh, S.-K.; Park, H.-S.; Lee, K.-N.; Hwang, S.H.; Choi, J.-I.; Kim, Y.-H. Different Responses of Left Atrium and Left Atrial Appendage to Radiofrequency Catheter Ablation of Atrial Fibrillation: A Follow Up MRI study. Sci. Rep. 2018, 8, 7871. [CrossRef]

10. Ouyang, F.; Tilz, R.; Chun, J.; Schmidt, B.; Wissner, E.; Zerm, T.; Neven, K.; Köktürk, B.; Konstantinidou, M.; Metzner, A.; et al. Long-Term Results of Catheter Ablation in Paroxysmal Atrial Fibrillation: Lessons from a 5-Year Follow-Up. Circulation 2010, 122, 2368-2377. [CrossRef]

11. Münkler, P.; Kröger, S.; Liosis, S.; Abdin, A.; Lyan, E.; Eitel, C.; Eitel, I.; Meyer, C.; Willems, S.; Heeger, C.-H.; et al. Ablation Index for Catheter Ablation of Atrial Fibrillation-Clinical Applicability and Comparison with Force-Time Integral. Circ. J. 2018, 82, 2722-2727. [CrossRef] [PubMed]

12. Verma, A.; Jiang, C.; Betts, T.R.; Chen, J.; Deisenhofer, I.; Mantovan, R.; Macle, L.; Morillo, C.; Haverkamp, W.; Weerasooriya, R.; et al. Approaches to Catheter Ablation for Persistent Atrial Fibrillation. N. Engl. J. Med. 2015, 372, 1812-1822. [CrossRef] [PubMed] 
13. Scherr, D.; Khairy, P.; Miyazaki, S.; Aurillac-Lavignolle, V.; Pascale, P.; Wilton, S.B.; Ramoul, K.; Komatsu, Y.; Roten, L.; Jadidi, A.; et al. Five-Year Outcome of Catheter Ablation of Persistent Atrial Fibrillation Using Termination of Atrial Fibrillation as a Procedural Endpoint. Circ. Arrhythm. Electrophysiol. 2015, 8, 18-24. [CrossRef] [PubMed]

14. Garvanski, I.; Simova, I.; Angelkov, L.; Matveev, M. Predictors of Recurrence of AF in Patients After Radiofrequency Ablation. Eur. Cardiol. Rev. 2019, 14, 165-168. [CrossRef]

15. Sultan, A.; Lüker, J.; Andresen, D.; Kuck, K.H.; Hoffmann, E.; Brachmann, J.; Hochadel, M.; Willems, S.; Eckardt, L.; Lewalter, T.; et al. Predictors of Atrial Fibrillation Recurrence after Catheter Ablation: Data from the German Ablation Registry. Sci. Rep. 2017, 7, 16678. [CrossRef]

16. Montefusco, A.; Biasco, L.; Blandino, A.; Cristoforetti, Y.; Scaglione, M.; Caponi, D.; Di Donna, P.; Boffano, C.; Cesarani, F.; Coin, D.; et al. Left atrial volume at MRI is the main determinant of outcome after pulmonary vein isolation plus linear lesion ablation for paroxysmal-persistent atrial fibrillation. J. Cardiovasc. Med. 2010, 11, 593-598. [CrossRef]

17. Tedrow, U.B.; Conen, D.; Ridker, P.M.; Cook, N.R.; Koplan, B.A.; Manson, J.E.; Buring, J.E.; Albert, C.M. The Long- and Short-Term Impact of Elevated Body Mass Index on the Risk of New Atrial Fibrillation. J. Am. Coll. Cardiol. 2010, 55, 2319-2327. [CrossRef]

18. Cha, Y.-M.; Friedman, P.A.; Asirvatham, S.J.; Shen, W.-K.; Munger, T.M.; Rea, R.F.; Brady, P.A.; Jahangir, A.; Monahan, K.H.; Hodge, D.O.; et al. Catheter Ablation for Atrial Fibrillation in Patients with Obesity. Circulation 2008, 117, 2583-2590. [CrossRef]

19. Baek, Y.-S.; Yang, P.; Kim, T.-H.; Uhm, J.; Kim, J.; Joung, B.; Lee, M.; Pak, H.-N. Delayed recurrence of atrial fibrillation 2 years after catheter ablation is associated with metabolic syndrome. Int. J. Cardiol. 2016, 223, 276-281. [CrossRef]

20. Wazni, O.M.; Marrouche, N.F.; Martin, D.O.; Verma, A.; Bhargava, M.; Saliba, W.; Bash, D.; Schweikert, R.; Brachmann, J.; Günther, J.; et al. Radiofrequency Ablation vs. Antiarrhythmic Drugs as First-line Treatment of Symptomatic Atrial Fibrillation. JAMA 2005, 293, 2634-2640. [CrossRef]

21. Akoum, N.; Daccarett, M.; McGann, C.; Segerson, N.; Vergara, G.; Kuppahally, S.; Badger, T.; Burgon, N.; Haslam, T.; Kholmovski, E.; et al. Atrial Fibrosis Helps Select the Appropriate Patient and Strategy in Catheter Ablation of Atrial Fibrillation: A DE-MRI Guided Approach. J. Cardiovasc. Electrophysiol. 2010, 22, 16-22. [CrossRef] [PubMed]

22. Kottkamp, H.; Schreiber, D.; Moser, F.; Rieger, A. Therapeutic Approaches to Atrial Fibrillation Ablation Targeting Atrial Fibrosis. JACC Clin. Electrophysiol. 2017, 3, 643-653. [CrossRef] [PubMed]

23. Ali, R.L.; Hakim, J.B.; Boyle, P.M.; Zahid, S.; Sivasambu, B.; Marine, J.E.; Calkins, H.; Trayanova, N.A.; Spragg, D.D. Arrhythmogenic propensity of the fibrotic substrate after atrial fibrillation ablation: A longitudinal study using magnetic resonance imaging-based atrial models. Cardiovasc. Res. 2019, 115, 1757-1765. [CrossRef]

24. Fochler, F.; Yamaguchi, T.; Kheirkahan, M.; Kholmovski, E.G.; Morris, A.K.; Marrouche, N.F. Late Gadolinium Enhancement Magnetic Resonance Imaging Guided Treatment of Post-Atrial Fibrillation Ablation Recurrent Arrhythmia. Circ. Arrhythm. Electrophysiol. 2019, 12, e007174. [CrossRef] [PubMed]

(C) 2020 by the authors. Licensee MDPI, Basel, Switzerland. This article is an open access article distributed under the terms and conditions of the Creative Commons Attribution (CC BY) license (http://creativecommons.org/licenses/by/4.0/). 